\title{
On the plane representation of the homaloidal surfaces which have a twisted cubic as multiple curve.
}

\author{
By Dr J. F. Tinto.
}

(Read 8th June 1917. Received 12th July 1917.)

\section{Introductory.}

The most important application of the rational point transformations between two spaces lies in the construction of algebraical surfaces possessing singularities of various kinds and the investigation of their properties.

When a plane is transformed by such a transformation into a rational algebraical surface (homaloid) the geometry of the surface is immediately derivable from the geometry of the plane.

This derivation is facilitated by the introduction of an additional step, viz., the discovery of the plane representation of lowest order. The rational space transformation of course supplies us with a plane representation of the surface, but in general it is not that of lowest order.

A plane section of the surface will have as corresponding curve on the plane which corresponds to the surface a curve having an order equal to that of the surface and possessing multiple points at the intersections of the $F$-curve system with the plane. We have to transform this curve by Cremona transfurmations till its order is the lowest possible.

The representation of certain quartic and quintic homaloids has been discussed analytically by Clebsch.* Amongst the surfaces treated by him are $(a)$ quartics with a single nodal line, $(b)$ ruled quartics with a double twisted cubic, (c) quintics with a double twisted cubic, $(d)$ quintics with two non-intersecting vodal lines. In the following paper the plane representation of a widely extended class of homaloidal surfaces having a twisted cubic,

* Irath. Ann., Bd. I. and II. 
either proper or degenerate, as curve of any order of multiplicity will be investigated. These surfaces may have also chords of the cubic as multiple lines. For the derivation of the surfaces by the method of rational point transformations the reader is referred to a paper on "The Three-Dimensional Transformations founded on the Twisted Cubic and its Chord System," (Proc. E. M. S., Vol. XXXIV., p. 133). It will be found that the surfaces of Clebsch are the simplest cases of the class referred to. The notation here employed is that of the paper quoted above.

I.

Surfaces with a proper twisted cubic as multiple curve.

First Series.

2. The series is doubly infinite, and the representative surface ${ }_{n} F_{n}$ is of order $(2 p+2) n-3$ and has a twisted cubic $\beth_{3}$ as curve of multiplicity $(p+1) u-2$. It has also $\alpha_{i}$ chords of $\Sigma_{3}$ as lines of multiplicity $i n$ where $\alpha_{i}$ and $i$ satisfy the equations

$$
\Sigma u_{i} i^{2}=p^{2}-1 \text { and } \Sigma \alpha_{i} i=3(p-1) \text {, }
$$

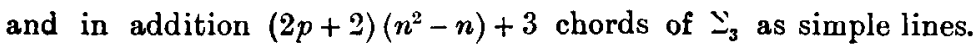
$p$ and $n$ are any positive integers.

To obtain the plane representation of lowest order we introduce the mechanism of the space transformation, the two spaces being in this case coincident. The $F^{\prime}$-systems, however, need not be supposed to be coincident.

Take an arbitrary plane $\pi$ and its corresponding surface ${ }_{p} F_{n}$. A plane section of ${ }_{p} F_{n}$ transforms into a curve on $\pi$ of order $(2 p+2) n-3$ with three nodes of order $(p+1) n-2$ at the points $A, B, C$, where $\dddot{Y}_{3}^{\prime}$ meets $\pi, \alpha_{i}$ nodes of order $i n$ and $3+(2 p+2)\left(u^{2}-u\right)$ simple points. Taking $A, B, C$ as $F$.points of a quadratic transformation, this curve becomes a curve of order $(p+1) n$ having $u_{i}$ nodes of order $i n$ and $3+(2 p+2)\left(n^{2}-n\right)$ simple points. A further transformation of this curve by a Cremona transformation of order $p$ having as $F$-system the $\alpha_{i}$ points yields a curve of order $(p+1) n$ of precisely similar nature. Hence the order $(p+1) n$ is the lowest possible.

\section{Plane representation of $\Sigma_{3}$.}

Corresponding to $\Sigma_{3}$ we get a surface of order $(4 p+4) n-(2 p+6)$ which meets $\pi$ in a curve of this order having $x$, nodes of order 
$i(2 n-1)$, nodes at $A, B, C$ of order $2(p+1) n-(p+4)$, and $3+(2 p+2)\left(n^{2}-n\right)$ double points.

This curve becomes, on applying the quadratic transformation, a curve of order $2(p+1) n-p$ having $\alpha_{i}$ nodes of order $i(2 n-1)$ and $3+(2 p+2)\left(n^{2}-n\right)$ double points. A further transformation of this curve by the Cremona transformation of order $p$ gives a curve of order $2(p+1) n-1$ having $a_{i}$ nodes of order $2 i n$ and $3+(2 p+2)\left(n^{2}-n\right)$ double points. No further diminution of this order is possible, and hence this curve is the required representation of $\Sigma_{3}$.

Again, corresponding to a point on $\Sigma_{3}$ we get a curve of order $(p+1) n-2$ lying on a rational ruled surface of order $2 p$ having $\alpha_{i}$ chords of $\Sigma_{3}$ as lines of multiplicity $i$. This surface meets $\pi$ in a curve of order $2 p$ having nodes of order $p$ at $A, B, C$ and $\alpha_{i}$ nodes of order $i$. Applying to this the above transformations, we obtain a straight line. Consequently, the points on $\pi$ which correspond to any point of $\Sigma_{3}$ form a group of $(p+1) n-2$ collinear points. The lines which contain these groups envelope a conic. For if a point describe $\Sigma_{3}$, the surfaces of order $2 p$ envelope a surface of order $4 p$ having $\Sigma_{3}$ as curve of multiplicity $2 p$ and the $\alpha_{1}$ lines as lines of multiplicity $2 i$.

This envelope is the correspondent of the quartic developable generated by the tangents to $\Sigma_{3}$. The curves of order $2 p$ on $\pi$ therefore envelope a curve of order $4 p$ having nodes of order $2 p$ at $A, B, C$ and $\alpha_{i}$ nodes of order $2 i$. Transforming this curve as before, we obtain a conic. This conic will be denoted by $C_{2}^{\prime}$.

\section{Plane representation of the multiple chords.}

The curve corresponding to a point on a multiple chord of order $i n$ is of order $i n$. It lies on a ruled surface of order $2 i$ having $\Sigma_{3}$ as curve of multiplicity $i$ and $\alpha_{i}$ chords of multiplicity $\beta_{i j}$ where $\sum_{j=1} i \beta_{i j}=p i$. Transforming the section of this surface by $\pi$, we obtain a point, viz., one of the multiple points of order $i n$.

Corresponding to the points of intersection of a multiple chord of order $i n$ with $\Sigma_{3}$ we get a degenerate curve of order $(p+1) n-2$ composed of a curve of order $(p+1-i) n-2$ and a curve of order $i n$. The collinear groups on $\pi$ corresponding to these points consist of $i n$ points coalescing at one of the $\alpha_{i}$ points and a group 
of $(p+1-i) n-2$ points whose line of collinearity passes through the $i$-point.

The $F$-point system on $\pi$ consequently consists of $\alpha_{i}$ points of order $i n$ (which will be referred to as the $i$-points) and $3+(2 p+2)\left(n^{2}-n\right)$ simple points.

\section{Systems of curves on ${ }_{p} F_{n}$.}

An arbitrary line on $\pi$ transforms into a rational curve of order $(p+1) n$ on ${ }_{p} F_{n}$ meeting $\Im_{3}$ in $2(p+1) n-1$ points. This curve is the intersection of a conicoid which contains $\Sigma_{3}$ with ${ }_{p} F_{n}$. The system is doubly infinite.

A rational curve of order $p$ on $\pi$ having $\alpha_{i}$ nodes of order $i$ also transforms into a curve of order $(p+1) n$, and the system is also doubly infinite. Any curve of the first system intersects a curve of the second in $p$ points. The curves of the first do not in general meet any of the multiple chords, whereas the curves of the second meet a chord of order $i n$ in $i$ points. Any two curves belonging to the same system intersect in one point.

Generally, a curve of order $l$ on $\pi$ which has an $i$-point as node of order $\gamma_{i}$ and nodes of orders $a, b, c, d$, etc., at the simple $F$-points transforms into a curve of order $l(p+1) n-\Sigma \alpha_{i} \gamma_{i} i n-\Sigma a$. In particular, a line through an $i$-point transforms into a curve of order $(p+1-i) n$, which meets the corresponding multiple chord in one point.

If a line touch the conic $C_{2}$, the corresponding curve has a node of order $(p+1) n-2$ on $\Sigma_{3}$. It also touches a fixed curve of order $2(p+1) n$, which corresponds to $C_{2}$. The latter curve is the intersection of the surface with the quartic developable of the tangents to $\Sigma_{3}$. This system of nodal curves is singly infinite.

A tangent to $C_{2}$ through a $i$-point transforms into a curve of order $(p+1-i) n$, having a node of order $(p+1-i) n-2$ at the intersection of the corresponding $i$-chord with $\Sigma_{3}$. The number of these curves is $2 \Sigma \alpha_{i}$.

Let the eurve on $\pi$ which represents $\Sigma_{3}$ be denoted by $C \Sigma_{3}$. A tangent to $C_{2}$, which also touches $C \Sigma_{3}$ at a point belonging to a collinear group of $(p+1) n-2$ points transforms into a curve having a node on $\Sigma_{3}$, at which two of the branches touch each other. Two of the sheets of the surface at this point have the same tangent plane, and such a point is a pinch point on $\Sigma_{3}$. 


\section{Pinch points on $\Sigma_{3}$.}

The number of pinch points on $\Sigma_{3}$ is not easy to determine directly.*

For a surface of order $2 m-1$ having $\Xi_{3}$ as curve of multiplicity $m-1$, but without multiple chords, I have found by an extension of the analytical method of Clebsch that the number of pinch points on $\Xi_{:}$is $2 m^{2}-8$. The presence of muitiple chords reduces this number.

The diminution in the number of common tangents to $C_{2}$ and $C_{s_{3}}$ due to the presence of an $i$-point of order $i n$ is $2 i n(i n-1)$. The total diminution is therefore $2 \alpha_{i}$ in $(i n-1)$ or

$$
2 n^{2}\left(p^{2}-1\right)-6 n(p-1) \text {. }
$$

The number of pinch points on $\Sigma_{3}$ is consequently

or

$$
\begin{gathered}
2[(p+1) n-1]^{2}-8-2 n^{2}\left(p^{2}-1\right)+6 n(p-1) \\
(4 p+4) n^{2}+2 n(p-5)-6 .
\end{gathered}
$$

7. Triple tangent planes.

A plane section of ${ }_{p} F_{n}$ through a simple chord of $\beth_{3}$ is repre. center on $\pi$ by a curve of order $(p+1) u$ having a node at one of the simple $F$-points. The planes of these sections are bitangent planes to the surfice, and we have consequently $3+(2 p+2)\left(n^{2}-n\right)$ pencils of such planes. In each pencil a finite number of planes will be triple tangent planes, and when this is the case the curve of order $(p+1) n$ will have an additional node. The number of curves of order $m$ belonging to a pencil and having $\lambda_{i}$ nodes of order $i$ which possess an additional node is $3(m-1)^{2}-\dot{\Sigma}(i-1)(3 i+1) \lambda_{i} \cdot \dagger$ Substituting for $m, \lambda$ and $i$, we get for the number of triple tangent planes through a simple chord

$$
(6 p+6) n^{2}-12 n-4+2 x_{i}
$$

The total number of such planes is therefore

$$
\left.[(-p+2)(n+-n)+3][6 p+6) n^{2}-12 n-4+2 x_{i}\right] .
$$

This number does not in general exhaust the triple tangent planes which the surface can possess. For particular surfaces

A direct determination is given in $5: 22$.

Nuether (Math. Ann., Bd. IIL., p. 223), and Cayley (Crelle, Bd. 63). lor a simple analytical proof see Proc. L. M. S., III., p. 14. 
of the scries a plane section of order $(2 p+2) n-3$ may be degenerate and composed of two curves which intersect in 3 points which do not lie on $\mathrm{Y}^{\prime}$, or on a multiple chord. Such planes are also triple tangent planes. Or again, the surface may have additional lines, though these will not be chords of $S_{i v}$, and through them it will be possible in general to describe a definite number of triple tangent planes.

\section{Nature of the F-system of points on $\pi$.}

Since the plane sections of ${ }_{p} F_{n}$ are triply infinite in number, it follows that the system of $F$-points on $\pi$, consisting of $\alpha_{i}$ points of order $i n$ and $3+(2 p+2)\left(n^{2}-n\right)$ simple points, must be such that the system of curves of order $(p+1) n$ through them is also triply infinite.

For values of $n>2$ this condition will not be satisfied by an arbitrary choice of the $F$-points.

The $F$-point system forms in this case what Cayley called a special system. Then if we put $p=1$ and $n=3$, we gret a nonic surface whose plane representation is of order 6 , and there are 27 simple $F$-points. Now, a sextic being deternined completely by 27 arbitrary points, it follows that the $27 F$-points are of a special kind. Instead of imposing 27 conditions on the sextic, they must in effect impose only 24. To find an explanation for this we refer again to the space transformation. For the particular case of the nonic surface we have a triply infinite number of such surfaces having $\Sigma_{3}$ as curve of multiplicity 4 , and containing an $F$-curve of order 24 . Hence on the plane $\pi$ we have a triply infinite system of curves of order 9 with nodes of 4 at $A, B, C$ and 24 simple points. Transforming by a quadratic transformation with $A, B, C$ as $F$-points, we obtain a system triply infinite of sextics through 27 points. We thus infer that it is possible through the $27 F$-points to pass a triply infinite system of sextics.

\section{Direct derivation of the plane representation of ${ }_{n} F_{n}$.}

The results contained in the previous articles may be obtained as follows.

Any point on ${ }_{p} F_{n}$ is connected with a point $Q$ of $\pi$ by means of the unique chord of $\searrow_{3}$ through $P$. The quadratic transformation with $A, B, C$ as $F$-points yields as the correspondent of $Q$ at a point 
$Q^{\prime} . \quad P$ and $Q^{\prime}$ are taken as corresponding points on ${ }_{p} F_{n}$ and $\pi$ respectively. The correspondence is evidently $(1,1)$.

A plane section of ${ }_{p} F_{n}$ becomes on $\pi$ a curve of order $(p+1) n$, having $\alpha_{i}$ nodes of order $i n$ and $3+(2 p+2)\left(n^{2}-n\right)$ simple points. If $H$ be any point on $\Sigma_{3}$, not on a multiple chord, we obtain, by taking the chords of $\Sigma_{3}$ through the points of the different sheets of the surface consecutive to $H$, a group of $(p+1) n-2$ points lying on a conic through $A, B, C$. These conics envelope a tricuspidal quartic with cusps at $A, B, C$. This quartic transforms into a conic touching the sides of the triangle $A, B, C$. The analytical method employed by Clebsch (Math. Ann., I.) in the case of the quintic is substantially identical with this.

A direct method such as this is, of course, only of limited application.

\section{Second Series.}

10. Another remarkable series of surfaces of the same type as the above is obtained by transforming a cubic surface which contains $\Sigma_{3}$ in space (1). Choosing 3 of its 6 lines which

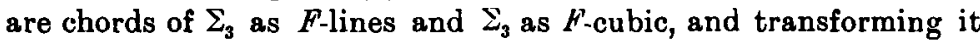
by a cubic transformation into a space $\left(1^{\prime}\right)$, we get a plane. Transforming the cubic into space (2), we get a surface of order $(2 p+2) n+2 p-3$ having $\Sigma_{3}$ as curve of multiplicity $(p+1) n+p-2$, $\alpha_{i}$ chords of order $i(n+1)$ and $6+(2 p+2)\left(n^{2}-1\right)$ simple chords. In what follows the results only will he given, the details of their derivation being omitted.

The plane representation of lowest order is of order $(p+1) n+p$. The $F$-point system on $\pi$ consists of $\alpha_{i}$ points of multiplicity $i(n+1)$ and $6+(2 p+2)\left(n^{2}-1\right)$ simple points. $\Sigma_{3}$ is represented by a curve of order $2(p+1) n+2 p-1$ having the $i$-points as points of order $2 i(n+1)$ and the simple points as double points.

The number of proper pinch points on $\Sigma_{3}$ is

$$
(4 p+4) n^{2}+n(6 p-6)+2 p-10 .
$$

The number of triple tangent planes through simple chords is

$$
\left[6+(2 p+2)\left(n^{2}-1\right)\right]\left[n^{2}(6 p+6)-18 n-6 p n+\Sigma \alpha_{i}-5\right] \text {. }
$$

The surfaces of the series are in order intermediate to those of the first. 


\section{General observations on the two series.}

The most general type of homaloid having $\Sigma_{3}$ as multiple curve is obtained by putting $p=1$. The two series can in this case be combined into a single one. The order of the representative surface is $2 m-1, \Sigma_{3}$ being of multiplicity $m-1$, where $m$ is any positive integer. There are no multiple chords, and the number of simple chords is $m^{2}+2$. The order of the plane representation is $m+1$. There are $2 m^{2}-8$ pinch points on $\Sigma_{3}$, and there are $\left(m^{2}+2\right)\left(3 m^{2}-7\right)$ triple tangent planes through simple chords of $\Sigma_{3}$.

Putting $m=2$ we get the well-known representation of the non-singular cubic surface.

Putting $m=3$ we obtain the results of Clebsch for the quintic. On the quintic there are 11 lines and 55 conics, and the planes of these conics are also triple tangent planes. The representative of $\Sigma_{3}$ in the case of the cubic is a unicursal curve of order 5 with 6 double points. In the case of the quintic it is a hyperelliptic curve of deficiency 4 and order 7 with 11 double points. For values of $m$ greater than 3 the curve which represents $\Sigma_{3}$ is connected with transcendents of Abelian type.

The presence of a multiple chord will restrict the generality of the surface and cause a diminution in the number of simple chords. Let the surface of order $2 m-i$ be supposed to possess a multiple chord of order $i$, then, since two curves of order $m+1$ on $\pi$ must intersect in $2 m-1$ points outwith the $F$-point system, we must have, if $v$ be the number of simple $F$-points,

$$
\begin{gathered}
(m+1)^{2}-i^{2}-v=2 m-1, \\
\nu=m^{2}+2-i^{2} .
\end{gathered}
$$

Thus a chord of order $i$ diminishes the number of simple chords by $i^{2}$.

II.

$\Sigma_{3}$ is degenerate and composed of a conic $\sigma_{2}$ and a line $\sigma_{1}$ which meets the conic.

First Series.

12. The representative surface is of order $(2 p+1) n-2, \sigma_{1}$ being of multiplicity $(p+1) n-2$ and $\sigma_{2}$ of multiplicity $n p-1$. There are $a_{i}$ chords of order $i n$, and $2+(2 p+1)\left(n^{2}-n\right)$ simple chords. 
The plane representation is again of order $(p+1) n$ The curve of order $(p+1) n$ corresponding to a plane section of the surface has a multiple point $a$ of order $n, \alpha_{i}$ points of order $i n$, and $2+(3 p+1)\left(n^{2}-n\right)$ simple points.

The curve $C \sigma_{1}$ which represents $\sigma_{1}$ is of order $(p+1) n-1$, and $C \sigma_{2}$ which represents $\sigma_{2}$ is of order $(p+1) n$.

On $C \sigma_{1}$ the point $a$ is of order $n-1$, and on $C \sigma_{2}$ of order $n+1$. The $i$-points are points of order $i n$, and the simple points simple points on each.

To the point a corresponds the residual intersection of the plane of the conic $\sigma_{2}$ with the surface, viz., a curve of order $n$. It is rational, and has a node of order $n-1$ at the point where $\sigma_{1}$ meets $\sigma_{2}$.

A line through $a$ transforms into a curve of order $p n$, which meets $\sigma_{1}$ in $p n$ points; it must consequently be a section through $\sigma_{1}$. The curve has a node of order $p n-1$ on $\sigma_{n}$.

The collinear group of $n-1$ points corresponding to a point on $\sigma_{2 .}$ lies on a line which envelopes $a$. The group of $(p+1) n-2$ points corresponding to a point of $\sigma_{1}$ lies on a line which envelopes a point $b$ on $C \sigma_{1}$. The line joining $a b$ meets $C \sigma_{1}$ and $C \sigma_{2}$ in $p n-1$ points which are groups for both. These points are fixed, and the line $a b$ corresponds to the section of the surface by a plane through $\sigma_{1}$ tangent to $\sigma_{2}$.

The conic $C_{2}$ is now degenerate and composed of two points.

\section{Pinch points on $\sigma_{1}$ and $\sigma_{2}$.}

The number of pinch points on $\sigma_{1}$ is equal to the number of tangents which can be drawn from $b$ to $C \sigma_{1}$. It is

$$
(2 p+1) n^{2}-3 n-2 \text {. }
$$

Similarly, the number of pinch points on $\sigma_{2}$ is the number of tangents which can be drawn from $a$ to $C \sigma_{2}$. This number is

$$
(2 p+1) n^{2}+2 p n-7 n-2 .
$$

The number of triple tangent planes through simple chords is

$$
\left[2+(2 p+1)\left(n^{2}-n\right)\right]\left[(6 p+3) n^{2}-10 n-p n+\Sigma \alpha_{i}-2\right] .^{*}
$$

- There is a reduction of $p n-1$ to be made from the number derived from the formula of $\$ 7$. This arises from the prosence of a degenerate curve in the pencil. 


\section{Second Series.}

14. The representative surface derived, as in $\$ 10$, is of order $(2 p+1) n+2 p-2$. It has $\alpha_{i}$ chords of order $i(n+1)$ and $5+(n-1)[2 p(n+1)+n+2]$ simple chords. $\sigma_{1}$ is of multiplicity $(p+1) n+p-2$, and $\sigma_{2}$ of multiplicity $n p+p-1$.

The plane representation is of order $(p+1) n+p$. The curves of order $(p+1) n+p$ bave, as before, a multiple point $a$ of order $n$.

$C \sigma_{1}$ is of order $(p+1) n+p-1$, and has $a$ for a point of order $n-1$. $C \sigma_{2}$ is of order $(p+1) n+p$, and has $a$ for a point of order $n+1$.

The pinch points on $\sigma_{1}$ number $(2 p+1) n^{2}+n(2 p-1)-4$. On $\sigma_{2}$ the number is $(2 p+1) n^{2}+n(4 p-5)+2 p-4$.

The number of triple tangent planes is $\left[(6 p+3) n^{2}+5 p n-4 n-p+\Sigma \alpha_{i}-5\right][5+(n-1)\{2 p(n+1)+n+2\}]$.

\section{Particular cases.}

If we put $p=1$, the orders of the above series become $3 n-2$ and $3 n$ respectively.

For $n=2$ we get from the first a quartic with a nodal line. From the above formulae the results obtained by Clebsch follow readily.

Putting $n=2$ in the second series, we get a sextic surface having $\sigma_{2}$ as triple line and $\sigma_{2}$ as double conic. The surface contains 15 simple chords. A plane section is represented by a quintic curve having a double point at $a$ and 15 simple points. $C \sigma_{1}$ is of order 4 , and has a simple point at $a . \quad C \sigma_{2}$ is of order 5, and has a triple point at $a$. The latter is hyperelliptic, and being of deficiency 3 , we should expect that $\sigma_{2}$ would have 8 pinch points on it. There are, in fact, 8 pinch points on $\sigma_{2}$ and 10 on $\sigma_{1}$. The lines joining $a$ to each of the 15 simple points give rise to conics on the surface. The correspondent of $a$ itself is a conic, so that there are 16 simple conics on the surface.

The number of triple tangent planes is $15 \times 32$ or 480 . The planes of the conics are quadruple tangent planes.

\section{Third Series.}

16. For this type of degenerate cubic a third series of surfaces exists. A conicoid through $\Sigma_{2}$ transforms into a surface 
of order $(2 p+1) n-1$, having $\Sigma_{2}$ as curve of multiplicity $(p+1) n-2$, together with $\alpha_{i}$ chords of multiplicity $i n$ and $2+(n-1)[(2 p+1) n+2]$ simple chords. The plane representation of sections of the surface is still of order $(p+1) n$. The curves of this order have a node of order $(n-1)$ at $a$, and $2+(n-1)[(2 p+1) n+2]$ simple points.

$C \sigma_{1}$ is of order $(p+1) n-1$ with $a$ of order $n-2 . C \sigma_{2}$ is of order $(p+1) n$ with $a$ of order $n$. The number of pinch points on $\sigma_{1}$ is $(3 p+1) n^{2}-n-6$, and the number on $\Sigma_{2}$ is $(2 p+1) n^{2}+n(2 p-5)$. The number of triple tangent planes is

$\left[(6 p+3) n^{2}-4 n-p n+\sum \alpha_{i}-9\right][2+(n-1)\{(2 p+1) n+2\}]$.

As particular cases we have the following : $p=2, n=1$ gives a quartic with a double conic. This surface was also discussed by Clebsch (Crelle, Bd. 68). $\quad p=1, n=2$ gives a quintic with a double line and double conic, the degenerate case of the quintic of $\$ 11$.

III.

$\Sigma_{3}$ is degenerate and composed of two non-intersecting lines $\sigma_{1}$ and $\sigma_{2}$, which are met by a third $\sigma_{3}$.

First Series.

17. The representative surfaces of order $2 n p-1$ having $\sigma_{1}$ and $\sigma_{2}$ as lines of multiplicity $n p-1$ and $\sigma_{3}$ of multiplicity $n(p-1)$. It possesses also $\alpha_{i}$ chords (of $\sigma_{1} \sigma_{2}$ ) of multiplicity $i n$ and $1+2 p\left(n^{2}-n\right)$ simple chords. The plane representation is of order $(p+1) n$, but there are two cases according as $p=1$ or $p>1$. For $p>1,(p+1) n$ is of the lowest possible order. The curves of this order on $\pi$ have two multiple points $a$ and $a^{\prime}$ of order $n$, $\alpha_{i} F$-points of order $i n$ and $1+2 p\left(n^{2}-n\right)$ simple points. Corresponding to each of the points $a, a^{\prime}$, we get a plane curve on the surface which is the residual intersection of the planes $\sigma_{1} \sigma_{3}, \sigma_{2} \sigma_{3}$ with the surface. These curves are rational and have each nodes of order $n-1$ at the point in which their plane intersects $\sigma_{1}$ or $\sigma_{2}$. $\sigma_{1}$ is represented by a curve of order $(p+1) n-1$, having $a$ as node of order $n-1$ and $a^{\prime}$ as node of order $n . \quad \sigma_{2}$ is represented by a curve of the same order, having $a$ as node of order $n$ and $a^{\prime}$ as node of order $n-1$. The lines of the collinear groups of $n p-1$ points corresponding to points on $\sigma_{1}$ envelope $a^{\prime}$, and similarly the lines of the groups for points on $\sigma_{2}$ envelope $a$. The number of pinch points on $\sigma_{1}$ and $\sigma_{2}$ is $2 p n^{2}-4 n$. 
The number of triple tangent planes through a simple chord is $\left[6 p n^{2}-2 p n-8 n+\Sigma \alpha_{i}+2\right]\left[1+2 p\left(n^{2}-n\right)\right]$, excluding the planes through $\sigma_{1}$ and $\sigma_{2}$, which are $(p n-1)$-ple tangent planes.

The line $a a^{\prime}$ represents the chord $\sigma_{\dot{s}}$.

For $p=1$ a further quadratic transformation is necessary, having $A B$ and one of the simple $F$-points as $F$-points. Applying this to the curve of order $(p+1) n$, we get a curve of order $2 n p-1$, having two nodes of order $p n-1$ and $2 p\left(n^{2}-n\right)$ simple points.

Putting $p=1$, we see that plane sections of the surface are represented by curves of order $2 n-1$, having 2 points of order $n-1$ and $2 n(n-1)$ simple points.

$\sigma_{1}\left(\sigma_{2}\right)$ is represented by a curve of order $2 n-2$, having $a\left(a^{\prime}\right)$ as node of order $n-2,(n-1)$.

The line $a a^{\prime}$ represents the simple chord corresponding to the point chosen in the quadratic transformation. The number of pinch points on $\sigma_{1}\left(\sigma_{2}\right)$ is $2 n^{2}-4 n$. The number of triple tangent planes is $[1+2 n(n-1)]\left[6 n^{2}-10 n+1\right]$.

\section{Second Series.}

18. The surfaces are of order $2 n p+2 p-1 . \quad \sigma_{1}$ and $\sigma_{2}$ are lines of multiplicity $n p+p-1$, and $\sigma_{3}$ is of multiplicity $n(p-1)+p$.

There are $\alpha_{i}$ chords of order $i(n+1)$ and $4+(n-1)[2 p(n+1)+2]$ simple chords.

The plane representation of lowest order for all cases is of order $(p+1) n+p$.

A plane section of the surface transforms into a curve of order $(p+1) n+p$, having two multiple points of order $n\left(a, a^{\prime}\right), \alpha_{i}$ points of order $i(n+1)$, and $4+(n-1)\{2 p(n+1)+2\}$ simple points.

$\sigma_{1}$ and $\sigma_{2}$ are represented by curves of order $(p+1) n+p-1$, having $a\left(a^{\prime}\right)$ as nodes of order $n-1(n) . \quad \sigma_{3}$ is represented by the line $a a^{\prime}$. The pinch points on $\sigma_{1}$ or $\sigma_{2}$ number $2 p n^{2}+2 n(p-1)-2$. The number of triple tangent planes is

$$
\left[6 p n^{2}+4 p n-2 n-2 p+\Sigma \alpha_{i}-1\right][4+(n-1)\{2 p(n+1)+2\}] \text {. }
$$

For $p=1$ the series is identical with the preceding, and may be obtained by putting in place of $n$ in it $n+1$.

\section{Particular cases.}

Putting $p=1, n=3$ in first series or $p=1, n=2$ in second, we obtain a quintic with two non-intersecting nodal lines. This 
surface has 13 lines and 26 conics. There are 6 pinch points on each of $\sigma_{1}$ and $\sigma_{2}$. There are also $13 \times 25=325$ triple tangent planes through simple chords.

$\sigma_{1} \sigma_{:}$are represented by quartics having $a\left(a^{\prime}\right)$ as points of order $l(2)$, and vice-versa. These quartics are of deficiency 2 , and hence we should expect 6 pinch points on each of $\sigma_{1}$ and $\sigma_{2}$.

These results agree with those obtained analytically by Clebsch.

Third Series.

20. As in the preceding case (II.), a third series is obtained by transforming a conicoid through $\sigma_{1}$ and $\sigma_{3}$. The surfaces thus obtained are of order $2 n p$ and have $\sigma_{1}$ as line of multiplicity $n p-1$ and $\sigma_{2}$ as line of multiplicity $n p . \quad \sigma_{3}$ is of multiplicity $n(p-1)+1$.

There are, as in the previous cases, $\alpha_{i}$ chords of order $i n$; the number of simple chords is $1+(n-1)(2 p n+2)$. The curves representing plane sections are of order $(p+1) n$ with nodes of order $n, n-1$ at $a, a^{\prime}$, together with $\alpha_{i}$ multiple points of order $i n$, and $1+(n-1)(2 p n+2)$ simple points.

$\sigma_{2}$ is represented by a curve of order $(p+1) n-1$, having nodes of order $n-1$ at $a$ and $a . \quad \sigma_{1}$ is represented by a curve of order $\left(p+l^{\circ}\right) n-1$, having nodes of order $n-2, n$ at $a$ and $a^{\prime}$ respectively. $\quad \sigma_{3}$ is represented by the line $a a^{\prime}$. The number of pinch points on $\sigma_{2}$ is $2 p n^{2}-2 n$, and the number on $\sigma_{1}$ is $2 p n^{2}-2 n-4$.

The triple tangent planes number

$$
[1+(n-1)(2 p n+2)]\left[6 p n^{2}-2 p n-2 n+\Sigma \alpha_{i}-4\right] .
$$

The following particular cases are of interest: $p_{F} 2, n=1$ gives quartic with two intersecting double lines and 16 simple lines, treated by Körndorfer (Math. Ann. III.). $p=1, n=3$ gives a sextic with a triple line, a double line, and 18 simple lines. $p=1$ gives the singly infinite series of order $2 n$, with a line of multiplicity $n$ and one of multiplicity $n-1$.

IV.

Rational ruled surfaces having $\Sigma_{3}$ as multiple curve.

21. We shall treat briefly the plane representation of such surfaces. 
They are obtained by transforming a conicoid through $\Sigma_{3}$ by the transformation $T_{2 p-1}^{\prime}$ (loc. cit.). The order is $2 p . \quad \Sigma_{3}$ is a curve of multiplicity $p$, and there are $\alpha_{i}$ chords of multiplicity $i$.

Any plane section of the surface is unicursal. The plane representation is obtained by transforming the conicoid into a plane in a space ( $\left(1^{\prime}\right)$.

A plane section of the surface transforms into a curve of order $p+1$, having a multiple point of order $p$ and one of order $1(a, b)$.

$\Sigma_{3}{ }^{\prime}$ is represented by a curve of order $p+2$, having $a$ as multiple point of order $p$ and $b$ as a double point. The deficiency of this curve is $(p-1)$. Corresponding to a multiple chord of order $i$ we get $i$ straight lines through $a$.

Any generator of the surface is represented by a line through $a$. In particular, the line $a b$ corresponds to a fixed point $B$ on the surface. To $a$ corresponds a curve of order $\mu$ on the surface, and to $b$ a fixed generator through $B$.

The group of points of order $p$ which corresponds to a point of $Y_{3}^{\prime}$ is not a collinear group. The curves representing $\Psi_{;}^{\prime}$ are all hyperelliptic in character, and in the general case $2 p$ tangents can be drawn from $a^{\prime}$ to the curve. There are consequently $2 p$ tangents to ${ }_{3}^{\prime}$, which are generators of the surface.

22. Synthetic investigation for the number of pinch points on the cubic $\mathbb{S}_{3}$ when the latter is a proper curve.

Consider the pencil of curves of order $(p+1) n$ which have a particular simple $F^{\prime}$-point on $\pi$ as double point. This pencil corresponds to the plane sections of the surface through a simple chord. The number of base points of the pencil is $(p+1)^{2} n^{2}$. Of these the $F$-points account for $4+\left[2+(2 p+2)\left(n^{2}-n\right)\right]+\sum \alpha_{i} i^{2} n^{2}$, leaving $(2 p+2) n-6$. These must be fixed points on $C_{\Sigma_{3}}$, and together with the double point they constitute two groups of $(p+1) n-2$ points corresponding to the two intersections of the simple chord with $\Sigma_{3}$.

Any curve of the pencil meets $C_{\Sigma_{3}}$ in a group of $(p+1) n-2$ variable collinear points. When the cutting plane through the simple chord meets $\bigsqcup_{2}$ in a pinch point, two of the above points of intersection will coincide. We have consequently to find the number of curves of the pencil which touch $C_{\Sigma_{3}}$. By applying 
the method of correspondence I find that the points of contact lie on a curve of order $4(p+1) n-4$, having a node of order 3 at each of the $2+(2 p+2)\left(n^{2}-n\right)$ simple $F$-points, a node of order 5 at the double $F$-point, a node of order 2 at each of the $(2 p+2) n-6$ fixed points of $C_{\Sigma_{3}}$, and a node of order $4 i n-1$ at each of the $i$-points. The number of proper intersections of this locus with $C_{\Sigma_{3}}$ is therefore given by

$$
\begin{gathered}
4[(p+1) n-1][2(p+1) n-1]-10-2[(2 p+2) n-6] \\
-6\left[2+(2 p+2)\left(n^{2}-n\right)\right]-\Sigma \alpha_{i} 2 i n(4 i n-1),
\end{gathered}
$$

giving, on reduction, the expression already found, viz.

$$
(4 p+4) n^{2}+2 n p-10 n-6 \text {. }
$$

This is the number of curves which touch $C_{\Sigma_{3}}$, and hence the number of pinch points on $\Sigma_{3}$.

For the second series of surfaces we obtain similarly the number $(4 p+4) n^{2}+n(6 p-6)+2 p-10$.

By putting $p=1$ in these expressions and combining, we find that for a surface of order $2 m-1$ without multiple chords the number of pinch points is $2 m^{2}-8$. This method is of very general application.

For the ruled surfaces we find that a pencil of planes through a generator is represented on $\pi$ by a pencil of curves of order $p$, having as base points a node of order $p$ at the $F$-point of order $p$, a simple point at the $F$-point of order 1 , and $2(p-1)$ fixed points on $C_{\Sigma_{3}}$. While the determination of the number of pinch points by the above method presents no difficulty, the number is in this case more easily determined by applying to the pencil and $C_{\Sigma_{3 ;}}$ a de Jonquiere's transformation of order $p$, having the $p$-node and the $2(p-1)$ fixed points as $F$-system.

The pencil of curves transforms into a pencil of lines through

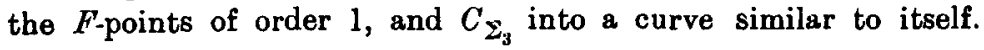
The number of tangents which can be drawn from the simple $F^{\prime}$-point to $C_{\Sigma_{3}}{ }^{\prime}$ is $4 p-4$, and this the number of pinch points on $\Sigma_{3}$. 